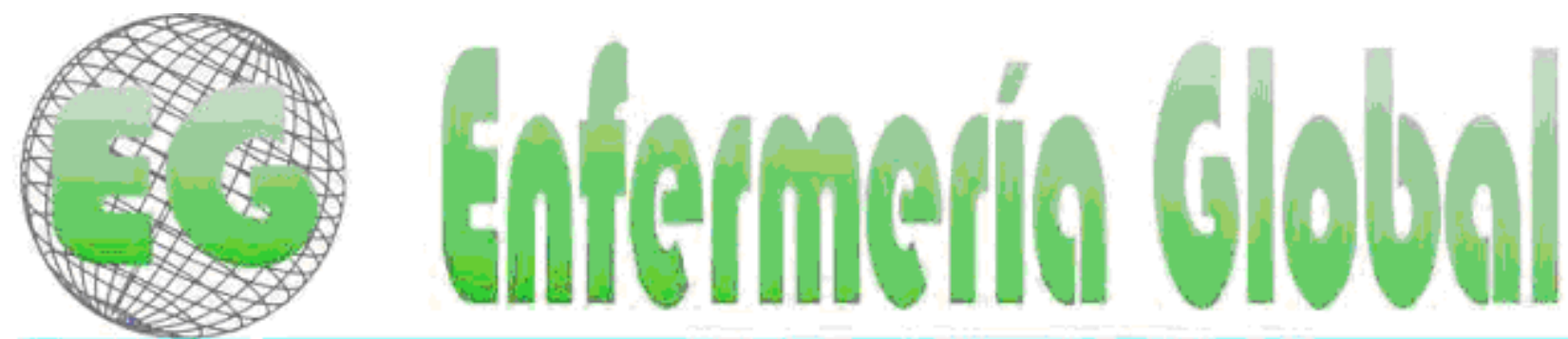

15SN 1696-6*5:

Revista electrônica cuatrimestral de Enfermeria

$\mathrm{N}^{\circ} 20$

Octubre 2010

www.um.es/eglobal/

\title{
CLÍNICA
}

\section{HÁBITOS ALIMENTARIOS DE LAS MUJERES EN RELACIÓN CON EL NIVEL DE CONOCIMIENTOS SOBRE EL CLIMATERIO}

EATING HABITS IN WOMEN WITH RELATION TO THEIR KNOWLEDGE LEVEL OF CLIMATERIC

${ }^{*}$ Moure Fernandez, L., "*Antolin Rodriguez, R., "**Puialto Duran, MJ, ${ }^{* * *}$ Salgado Alvarez, C.

*Unidad de Docencia y Formación continuada. **Escuela Universitaria de Enfermería. Hospital do Meixoeiro. ***Unidad Hospitalización planta $1^{\text {a }}$ Hospital Xeral ^. Complexo Hospitalario Universitario Vigo (CHUVI)

\begin{abstract}
(Financiado dentro del Programa de Promoción de la Investigación Biomédica y en Ciencias de la Salud para la realización de estudios de investigaciones sobre Evaluación de Tecnologías Sanitarias e Investigación en Servicios de Salud, en el Marco del Plan Nacional i+D+l 2004-2007 (PI07/90466), desarrollado en el año 2008.)
\end{abstract}

Palabras clave: Climaterio; menopausia; hábitos alimentación; conocimientos

Keywords: Climacteric, Menopause, Eating Habits, and Knowledge.

\section{RESUMEN}

Objetivos: Conocer los comportamientos relacionados con la alimentación y hábitos tóxicos de las mujeres de edad comprendida entre 30-60 años del área sanitaria de Vigo.

Averiguar si las mujeres menopáusicas tienen comportamientos más saludables que las no menopáusicas.Identificar si alguna de las variables sociodemográficas influye en los comportamientos sobre la alimentación y hábitos tóxicos. Determinar si las mujeres con conocimientos altos-muy altos tienen comportamientos adecuados

Métodos: Estudio comparativo, observacional, transversal, realizado a 425 mujeres de 30-60 años. Se utilizó un cuadernillo con dos cuestionarios autocumplimentados, uno de conocimientos sobre la menopausia y otro sobre los comportamientos relacionados con la alimentación en la etapa del climaterio, incluidas las variables sociodemográficas. El análisis de los datos se realizó mediante SPSS 14.0.

Resultados: Las mujeres de nuestra muestra tienen conocimientos altos-muy altos en un $74,3 \%$ y unos comportamientos que no se correlacionan con el nivel de conocimientos.

Conclusiones: Las mujeres conocen qué es lo que deben realizar para mantener una vida saludable, pero a la hora de ponerlo en práctica no lo hacen mayoritariamente. Consideramos necesaria la implantación en los Centros de Salud, de un programa coordinado por el equipo de 
salud, dirigido a aplicar los conocimientos que han demostrado poseer las mujeres a los comportamientos diarios, con el objetivo de alcanzar una mayor calidad de vida. Este estudio forma parte de una investigación más amplia realizada sobre los "Conocimientos y comportamientos relacionados con el climaterio de las mujeres de 30-60 años del Área Sanitaria de Vigo" becado por el Fondo de Investigaciones Sanitarias (2007).

\section{ABSTRACT}

Objectives: Knowing the behaviors related to food and toxic habits of women aged $30-60$ years in the health area of Vigo.

Determine whether menopausal women have healthier behaviors than non menopausal ones. Identify whether any of the socio-demographic variables has an influence on feeding habits or toxic behaviors. Determine whether women with high-very high knowledge have proper habits.

Methods: Comparative, observational cross study made of 425 women between 30 and 60 years of age. We used a booklet with personally answered questionnaires, one about menopause and the other about feeding behaviors during the climacteric stage, which includes socio-demographic variables. Data analysis was carried out using SPSS 14.0.

Results: A result of $74.3 \%$ of the women in the sample has a high/very high level of knowledge but behaviors that do not correlate with this.

Conclusions: Women know what they must do to maintain a healthy life, but the majority does not put this into practice. We think it is necessary to introduce in health centers a program about the climacteric stage, coordinated by nurses and midwives and focused on applying to daily life the knowledge that women already have, with the aim of helping them to achieve a better quality of life.

This study is part of a wider research on "Knowledge and behaviors related to climacteric stage in women between 30 and 60 years of age in the health area of Vigo sponsored by the "Fondo de Investigaciones Sanitarias" (Spanish Health Research Fund) in 2007.

\section{INTRODUCCIÓN}

El climaterio constituye una etapa de la vida de la mujer en la que se produce la desaparición de la ovulación y la disminución en la producción de estrógenos y progesterona ${ }^{1}$. El acontecimiento central del climaterio, lo constituye la menopausia, es decir, la ausencia definitiva de las reglas.

El número de mujeres en edad climatérica crece de día en día; en el año 2008, en España 8.436.336 mujeres estaban en periodo climatérico o postmenopáusico (45 o más años), con una expectativa de vida de más de 30 años$^{2}$. El 44,4 \% de las mujeres gallegas están en situación de menopausia, a la que llegan, por término medio a los 49,2 años ${ }^{3}$.

Como señala Rozenbaum ${ }^{4}$ el grupo de las mujeres menopáusicas se ha convertido en un importante problema de salud pública por múltiples razones: demográficas, sociológicas, fisiológicas, médicas, económicas y humanas. La $\mathrm{OMS}^{5}$ informaba a las autoridades sanitarias sobre las consecuencias del rápido aumento de mujeres postmenopáusicas, previsible para el 2030 y recomendaba la preparación de los servicios de salud y de las actividades educativas y de promoción adecuadas para atender las necesidades de salud de las mujeres en esa, cada vez, más amplia etapa de su vida.

Se ha demostrado que la sintomatología y signología propia del climaterio y la menopausia, no es una experiencia similar universal, sino que está condicionada a factores socioculturales $^{6}$. Asimismo, se ha demostrado que los cambios psicológicos, están relacionados con los síntomas vasomotores y con factores socioculturales, antes que con una respuesta a los cambios hormonales del climaterio ${ }^{7}$. 
Los estudios españoles revisados ${ }^{8-10}$ muestran que los conocimientos que las mujeres poseen sobre hábitos de salud, son incompletos o están poco fundamentados.

El desconocimiento y la desorientación que muchas mujeres experimentan en esta etapa es, con toda probabilidad, uno de los factores que más influyen en la vivencia negativa de la misma y en el déficit de autocuidados que se aprecia en una amplia mayoría de ellas ${ }^{8}$.

Según un estudio del Centro de Información de la Menopausia ${ }^{11}$, la falta de información sobre qué hacer para prevenir las complicaciones de la menopausia, es manifiesta en la mayoría de las mujeres.

Concretamente, en el estudio de Contreras $^{10}$, las mujeres piden más información antes de llegar a esta etapa, información que solicitan a los profesionales y que estos deben proporcionarles. También Arnedillo ${ }^{12}$ encontró que el $98 \%$ de las mujeres cree importante que se las informe antes de llegar a la menopausia y que al $96 \%$ le gustaría recibir algún tipo de información o consejo por parte de los profesionales sanitarios.

Los conocimientos que la mujer necesita para poder enfrentar con éxito el climaterio deben ser unos conocimientos enfocados a generar en ella actitudes y comportamientos saludables y a capacitarla para desarrollar prácticas de autocuidados preventivos y de mejora de su salud. No podemos olvidar que la ausencia de una ingesta adecuada es uno de los factores predisponentes para padecer enfermedad cardiovascular y osteoporosis y que, con prácticas saludables, se podrían prevenir esta última en un $90 \%{ }^{13}$.

Como señalan múltiples estudios sobre la menopausia ${ }^{8,10}$, para que la educación para la salud pueda ser eficaz, es necesario averiguar los conocimientos previos que tiene la población, para ajustar la planificación y la acción a las características y necesidades cognitivas, actitudinales y comportamentales de la población a la que va dirigida la intervención. Conocer sus preconcepciones y actitudes ante el tema, sus hábitos y comportamientos nocivos, etc., es el referente obligado a cualquier toma de decisión.

Nuestra hipótesis de trabajo ha sido que: las mujeres con un nivel de conocimientos altos/muy altos tienen comportamientos adecuados, para ello nos fijamos los siguientes objetivos:

- Conocer los comportamientos relacionados con la alimentación y hábitos tóxicos de las mujeres de edad comprendida entre 30-60 años del área sanitaria de Vigo.

- Averiguar si las mujeres menopáusicas tienen comportamientos, en cuanto a la alimentación, más saludables que las no menopáusicas.

- Identificar si alguna de las variables sociodemográficas, influye en los comportamientos sobre la alimentación y hábitos tóxicos

- Determinar si las mujeres con conocimientos altos/muy altos tienen comportamientos alimentarios adecuados

\section{MÉTODOS}

Estudio comparativo observacional transversal, realizado en mujeres de 30-60 años, residentes en el área sanitaria de Vigo.

Para el cálculo del tamaño muestral, se consideró el peor de los supuestos $(p=q=50)$, un nivel de confianza del $95 \%$ y una de $p=0,05$, seleccionando una muestra formada por 384 mujeres. Aumentamos a 425(un 10\% más) para contrarrestar las posibles pérdidas. 
Para la recogida de datos utilizamos dos cuestionarios presentados en un único cuadernillo: uno sobre comportamientos alimentarios, preparación de alimentos, distribución, frecuencia de la ingesta y hábitos tóxicos (Anexo1) utilizado por López Santos ${ }^{14}$, del cual eliminamos 68 ítems, porque nuestro estudio estaba dirigido a las conductas alimentarías y hábitos tóxicos, quedando constituido por 70 preguntas, y otro de "conocimientos sobre la menopausia" validado por García Padilla ${ }^{15}$ y utilizado en el estudio del mismo autor ${ }^{11}$, que consta de 56 preguntas dicotómicas. (Anexo 1)

Criterios de inclusión: Mujer, entre 30-60 años, comprender la lengua castellana y/o gallega y que acepte cumplimentar los cuestionarios.

Criterios de exclusión: Que no cumplimenten la edad.

Los cuadernillos fueron entregados y recogidos por las enfermeras durante los meses de Enero a Marzo del 2008 a las mujeres que, como acompañantes o para propia consulta, se encontraban en las salas de espera de los centros de salud del área sanitaria de Vigo, y que cumplían los criterios de inclusión. Los centros fueron escogidos contemplando la distribución de la población en el área sanitaria (semirural, rural, urbana).

Antes de entregar los cuestionarios se solicitó el consentimiento, garantizando en todo momento el anonimato.

Las variables del estudio fueron las incluidas en los cuestionarios y las variables sociodemográficas: edad, estado civil, nivel de instrucción, lugar de residencia, número de hijos y estado menopáusico.

Los datos se analizaron mediante el SPSS 14.0. La descripción de las variables cuantitativas se realizó con la media, desviación estandar(SD) e intervalo de confianza(IC), y en las cualitativas con el porcentaje. La comparación de variables cualitativas se realizó mediante el Chi²; las variables numéricas se compararon mediante t de Student o análisis de varianza en función de los grupos de comparación (2 ó más).

Como estrategia de búsqueda se utilizó: [Climacteric and behavior and eating habits] [climacteric and knowledge], en las bases de datos Medline, Scielo, Cuiden, Cinhal, Bdie. Los límites fueron: últimos 5 años, mujeres, 30-60 años, mediante revisiones indirectas se reseñan artículos de fechas anteriores que resultaron de interés

\section{RESULTADOS}

Se entregaron 425 encuestas, de las cuales se dieron por válidas en el apartado del cuestionario sobre conocimientos $377(88,70 \%)$

En el apartado sobre comportamientos, y con el fin de conseguir una pérdida mínima de información, se aceptaron todas las respuestas obtenidas en cada una de las preguntas de aquellas encuestas consideradas válidas en el apartado de conocimientos.

La media de edad de las mujeres fue de 45,99 con un IC de 45,14 - 46,85 y una SD de 8,47. Las mujeres consideradas preclimatéricas con edad comprendida entre 30-44 años representaron un 43,8\%(165) y las climatéricas con edad entre 45-60 años un 56,2\%(212). 
Un $37 \%(138)$, de las mujeres encuestadas, manifestaba que se le había retirado la regla siendo la retirada de forma natural en el $80 \%$ de los casos (112) pero solamente un $22,5 \%(85)$ había llegado a la menopausia.

Un $15,8 \%$ (59) vivía en zona rural, un $24,4 \%$ (91) en zona semirural y un $59,8 \%$ en zona urbana(59,8\%).

Estaban casadas el $67,1 \%$ y vivían en pareja un $75,6 \%$.

El $44,3 \%$ tenia dos hijos, el 19,6\% uno, siendo el IC de 1,62-1,40 ( $\bar{X}=1,50 ; S D=1,07)$

En el nivel de estudios destacar que un 37\%(137) tenía estudios universitarios.

\section{Comportamientos alimentarios}

En nuestro estudio hemos equiparado el número de tomas al número de raciones, ya que desconocemos la cantidad exacta de comida que ingieren en cada toma, al no estar este dato especificado en el cuestionario.

En relación a la frecuencia de consumo de los grupos básicos de alimentos que deben tomar las mujeres, hemos seguido la escala SENC (Sociedad Española de Nutrición Comunitaria) ${ }^{16}$ del 2001 con alguna modificación en la agrupación de los alimentos ${ }^{17}$, que indica las raciones de alimentos que se consideran saludables para la población en general, ya que la alimentación de la mujer en el climaterio no debe ser diferente a la de la población general.

En relación a los alimentos ricos en calcio, referían tomar yogures todos los días un $37,4 \%(138)$ de las mujeres y más de una vez al día un 17,8\%(66). En cuanto a la cantidad de leche que tomaban diariamente, un $43 \%(150)$ decía tomar dos o tres vasos y un 10,9\% (38) menos de un vaso al día.

El 53\%(169) manifestaba ingerir el aporte adecuado de lácteos (leche, yogures, queso fresco, queso curado, natillas) ${ }^{17}$ y únicamente un $13 \%$ (41) tomaba una cantidad mayor.

En relación al consumo de cereales (arroz, pasta, maíz): un 29,6\%(110) los tomaba una vez al día, un 52,5\%(195) lo hacía alguna vez a la semana, y un 4,8\%(18) raras veces.

Acerca de la frecuencia con que consumían patatas: un 32,2\%(119) lo hacía una vez al día, un $45 \%(166)$ alguna vez a la semana y un $12,2 \%(45)$ raras veces.

Respecto a la ingesta de pan resaltar que un 10,1\% (37) tomaba diariamente el equivalente a dos vienas-bollos. El tipo de pan que comían habitualmente era blanco en un 46,2\%(174) aunque un $27,6 \%(104)$ alternaba el pan blanco con el integral.

Reflejar que solamente el 10,5\%(34) de las mujeres tomaba un aporte adecuado de farináceos (cereales, patata, pan, y legumbres), resultando que en el $89,2 \%(290)$ de los casos la ingesta fue inferior a la recomendada.

El pescado azul era consumido alguna vez a la semana por un $80,6 \%(303)$ y en un $15,4 \%(58)$ de los casos raras veces o nunca. En cuanto al pescado blanco un $87,7 \%(329)$ lo consumía alguna vez a la semana. 
En relación a la frecuencia con que tomaban distintos tipos de carne destacar que: un $83,3 \%(312)$ consumía carne de ave alguna vez por semana, un 14,4\%(54) tomaba raras veces o nunca carne de ternera o cordero, y un 40\%(150) consumía carne de cerdo raras veces o nunca.

[Tabla I Tipos de carnes y embutidos]

\begin{tabular}{|c|c|c|c|c|c|c|c|c|}
\hline \multicolumn{9}{|c|}{ Tabla I Tipos de carnes y embutidos } \\
\hline & \multicolumn{2}{|c|}{ Ave } & \multicolumn{2}{|c|}{ Terneraloordero } & \multicolumn{2}{|c|}{ Cerdo } & \multicolumn{2}{|c|}{ Embutidos } \\
\hline & N & $x$ & N & $x$ & $N$ & $x$ & N & $x$ \\
\hline Mas de una vez al da & 3 & 8 & 2 & 0,5 & 2 & 0,5 & 4 & 1,1 \\
\hline Una vez al dia & 16 & 4,3 & 11 & 2,9 & 8 & 2,2 & 68 & 18,3 \\
\hline Aguna vez a la semana & 314 & 83.7 & 307 & 82,1 & 212 & 57,3 & 163 & 43,8 \\
\hline Raras veces & 35 & 9,3 & 49 & 13,1 & 123 & 33,2 & 110 & 29,6 \\
\hline Nunca & 7 & 1,9 & 5 & 1,3 & 25 & 6.8 & 27 & 7,3 \\
\hline
\end{tabular}

Manifestaron consumir embutido una vez al día un 18,3\%(68).

Un 5,3\%(20) respondió que tomaba huevos una vez al día.

La ingesta de proteínas adecuada (carnes, pescados, huevos y embutidos) resultó deficitaria en un $70,3 \%(249)$ de la muestra, excesiva en un $23,7 \%$ (84) y correcta en un $6 \%(21)$.

Tomaban las raciones adecuadas de verduras un $57,1 \%(205)$.

De las mujeres de nuestra muestra, tomaban más de una vez al día kiwis y ciruelas un $8,1 \%$ (30), una vez al día un $28,6 \%$ (106) y otras frutas un $30,7 \%$ (115) en el primer caso y un $71,2 \%(267)$ en el segundo caso.

El consumo de frutas adecuado fue cumplido por el $29,1 \%(104)$ de las mujeres. 
La ingesta de grasas considerada saludable era consumida por un $7,4 \%(25)$ y un $64,7 \%(219)$ dijo consumir grasas raramente o nunca.

El $74,1 \%(169)$ de las encuestadas tomaban las raciones consideradas adecuadas en relación a los dulces y refrescos y a la hora de tomar dulces el 60,5\%(228) decía consumirlos caseros (hechos con una grasa adecuada) ${ }^{18,19}$ y únicamente un $9,8 \%(37)$ los ingería de fabricación industrial (que utilizan grasas saturadas y trans) $)^{18,19}$

En cuanto a la forma de cocinar los alimentos: un $46,6 \%(174)$ ha manifestado que no tomaba fritos o que lo hacía pocas veces, un $57,9 \%$ (216) tomaba los alimentos cocidos con bastante o mucha frecuencia y los consumían asados un 40,3\%(149).

Los alimentos en conserva los tomaban con mucha o bastante frecuencia un $4,3 \%(16)$ y un $64,2 \%(235)$ no solía tomar precocinados.

Manifestaba tomar las verduras y las frutas crudas con bastante frecuencia el 75,9\% (280) de las mujeres.

Referían no tomar picantes el $63,6 \%(235)$ y no tomar los alimentos muy calientes el $44,7 \%(165)$.

Un $93,1 \%(351)$ cocinaba con aceite de oliva, un $22,8 \%(86)$ con girasol, y un $1,9 \%(7)$ con mantequilla.

En relación al consumo de agua, se encontró que, durante el verano, un 43,5\%(160) ingería entre un litro y litro y medio y un $29,6 \%$ (109) entre litro y medio y dos litros, variando el consumo durante el resto del año: en el primer caso al $47 \%(173)$ y en el segundo al $13 \%(48)$.

En cuanto a las cantidades de alimentos y su forma de distribución durante el día los resultados fueron que: un $55,7 \%(209)$ tomaba un desayuno moderado, un $60,4 \%$ (218) no tomaba aperitivo, un $66 \%$ (246) tomaba una comida moderada, un $40,1 \%$ (147) no tomaba merienda, un $58 \%(217)$ tomaba una cena moderada, y un $32,4 \%(121)$ escasa, no acostumbraba a tapear un $59,9 \%(219)$.

Considerados como "hábitos tóxicos" el consumo de alcohol, tabaco, café y/o té, hemos encontrado que un $57,6 \%$ (201) de la muestra nunca consumía alcohol o lo hacía en raras ocasiones. En cuanto al tipo de alcohol consumido el 98\% (348) nunca consumía licores o lo hacía en raras ocasiones; la cerveza y el vino eran consumidos varias veces por semana en un $18 \%(65)$ y en un $15,7 \%(57)$ respectivamente.

No había fumado nunca o lo habían dejado hacía más de un año el 63\% (228) de las encuestadas.

El café o té, era consumido más de dos veces al día por un 44,1\%(162) de las mujeres. 
[Tabla Il. Consumo café y/o té ]

\begin{tabular}{|l|c|c|}
\hline \multicolumn{3}{|c|}{ Tabla II Consumo café yo té } \\
\hline & N & \\
& \multicolumn{2}{|c|}{$\%$} \\
\hline Más de tres veces da & 53 & 14.4 \\
De dos a tres veces & 109 & 29.7 \\
Una vez al dia & 111 & 29.4 \\
Varias veces semana & 25 & 6.8 \\
Raras veces & 25 & 6.8 \\
Nunca & 44 & 12 \\
& & \\
& & \\
\hline
\end{tabular}

Las mujeres que estaban en la menopausia $22,5 \%(85)$ no tenían más comportamientos saludables que las no menopáusicas, la comparación ha resultado no significativa en: calcio, farináceos, proteínas, frutas, verduras, grasas, alcohol y tabaco. El consumo de dulces y refrescos ha sido significativo $(p=0,036)(1 \mathrm{gl})$. 
[Tabla III Comportamientos alimentarios en función de los conocimientos y del estado menopáusico]

Tabla III Comportamientos alimentarios en función de los conocimientos y del estado menopáusico

\begin{tabular}{|c|c|c|c|c|}
\hline \multirow[t]{2}{*}{ Alimentos } & \multicolumn{2}{|c|}{$\begin{array}{l}\text { Conocimientos } \\
\text { altosimuy altos }\end{array}$} & \multicolumn{2}{|l|}{ Menopausia } \\
\hline & $P$ & $\mathrm{GL}$ & $P$ & GL \\
\hline $\begin{array}{l}\text { Calcio } \\
\text { Farináceos } \\
\text { Proteinas } \\
\text { Frutas } \\
\text { Verduras } \\
\text { Grasas } \\
\text { Dulcesyy refrescos } \\
\text { Acoholes } \\
\text { Tabaco }\end{array}$ & $\begin{array}{l}0,68 \\
0,74 \\
0,88 \\
0,62 \\
0,001 \\
0,34 \\
0,07 \\
0,07 \\
0,63\end{array}$ & $\begin{array}{l}1 \\
1 \\
1 \\
1 \\
1 \\
1 \\
1 \\
1 \\
1\end{array}$ & $\begin{array}{l}0,65 \\
0,24 \\
0,47 \\
0,51 \\
0,78 \\
0,06 \\
0,03 \\
0,34 \\
0,50\end{array}$ & $\begin{array}{l}1 \\
1 \\
1 \\
1 \\
1 \\
1 \\
1 \\
1 \\
1 \\
1\end{array}$ \\
\hline
\end{tabular}

En cuanto a la posible asociación de las variables sociodemográficas con los comportamientos sobre el consumo de: calcio, farináceos, proteínas, frutas, verduras, grasas, dulces y refrescos, alcohol, tabaco, hemos encontrado que con la edad y el estado civil no hay relación. Al valorar el área de residencia con las anteriores variables vemos que: entre rural y semirural, y rural y urbano, y el consumo de farináceos, es significativo en el primer caso con una $(p=0,04)(2 \mathrm{gl})$ y en el segundo $(p=0,01)(2 \mathrm{gl})$, en cuanto al nivel de estudios encontramos relación: entre los farináceos $(p=0,048)(3 \mathrm{gl})$ y también con el tabaco $(p=0,009)(3 \mathrm{gl})$ en el resto de los consumos no hemos encontrado significación estadística.

El valor medio de conocimientos de las mujeres del estudio fue de 40,88, con un IC de 39,90 - 41,86 en una escala de medida de 0-56 puntos. Destacar que el $74,3 \%(280)$ de las respuestas correspondió a conocimientos altos y muy altos

Las mujeres con conocimientos altos/muy altos, no tenían comportamientos adecuados en relación al consumo de: calcio, farináceos, proteínas, frutas, grasas, dulces y refrescos, en cuanto a las verduras es significativo con una $(p=0,001)(1 \mathrm{gl})$.

La comparación entre el consumo de tabaco y el nivel de conocimientos altos/muy altos no tiene relevancia estadística $(\mathrm{p}=0,635)(1 \mathrm{gl})$ 


\section{DISCUSIÓN}

La bibliografía consultada ${ }^{8,10,20-23}$ asocia comportamientos alimentarios inadecuados con un nivel de conocimientos bajo, mientras que las mujeres de nuestro estudio, con un nivel de conocimientos alto/muy alto, no tienen los comportamientos alimentarios adecuados como cabría esperar, exceptuando en la ingesta de verduras. Únicamente las variables sociodemográficas, área de residencia y nivel de estudios han influido en algún comportamiento, así como el estado menopáusico que solo es significativo en cuanto a la toma de dulces y refrescos.

Nuestros datos parecen sugerir que los comportamientos inadecuados no están ligados a conocimientos deficientes sino a un posible problema de actitud debido tal vez a la dificultad de modificar conductas en la edad adulta.

En cuanto a los diferentes consumos de alimentos encontramos que la ingesta de proteínas es deficitaria en casi las tres cuartas partes de nuestra muestra, lo que no concuerda con la "enquisa sobre os hábitos alimentarios da población adulta galega, 2007"24 en la que el aporte de proteínas en la dieta resultó ser excesivo, esto quizás podría explicarse porque nuestra población está formada exclusivamente por mujeres.

El hecho de que en un $90 \%$ de la muestra el consumo de farináceos haya sido inferior a la recomendada para una dieta saludable, que un $64 \%$ afirmara consumir "raramente o nunca" grasas, que solamente un $10 \%$ de los dulces consumidos fueran de fabricación industrial unido a que más de la mitad de las mujeres tomaran dosis adecuadas de verduras, nos lleva a pensar que es innegable que vivimos en una sociedad en la que se da mucha importancia al aspecto físico, vivimos en una época en la que se prefieren los cuerpos delgados y estilizados, haciendo que exista una gran preocupación por el cuidado del cuerpo y de la alimentación y en la que el mensaje publicitario es: "estar sana es estar delgada" difundiéndose la idea explícita o implícitamente de que tener una figura delgada es sinónimo de éxito y esfuerzo.

Nos parece muy positivo el que un 53\% tome el aporte adecuado de lácteos, teniendo en cuenta que la capacidad para absorber calcio disminuye con la edad, debiéndose tal vez a las campañas preventivas frente a la osteoporosis, en las que se hace hincapié en una ingesta suficiente de calcio con la dieta o mediante suplementos ${ }^{25}$ y a que es una medida preventiva que no requiere tanto esfuerzo como el ejercicio físico o el control de la dieta.

Por todo ello consideramos necesaria la implantación en los Centros de Salud de un programa coordinado por el equipo de salud, dirigido a aplicar los conocimientos que han demostrado poseer nuestras mujeres, a sus comportamientos diarios, con el fin de lograr una mayor calidad de vida en esta etapa de cambios físicos y psicológicos, que tanta inseguridad produce a las mujeres.

A la hora de realizar el estudio, encontramos ciertas limitaciones: por un lado el posible sesgo de selección al captar a las mujeres únicamente en los centros de salud, y por otro que en la encuesta sólo se indicaba las veces al día que tomaban los alimentos, pero no las cantidades en cada una de las tomas, por lo que podría darse la circunstancia de que la cantidad de cada alimento fuera ingerido de forma excesiva en una sola toma. A pesar de ello, consideramos que los resultados de nuestro estudio pueden ser útiles para posteriores investigaciones sobre el tema. 
Nuestro agradecimiento a todas las mujeres de los Centros de Atención Primaria que han contribuido con sus contestaciones a la elaboración de este estudio. A Victor del Campo, por su inestimable apoyo en la metodología y a las enfermeras/os de los distintos Centros de Salud que han participado en la distribución de los cuestionarios: Alvarez A; Alvarez C; Amorin F; Bello M; Bonete C; Carracelas E; Chao MR; Corbillon A; Costela MT; Cabanelas E; Diaz C; Domínguez R; Ferreiro P; Ferro A; Fuentes M; Gándara MN; García E; Gómez R; Gómez R; González E; González J; Lago L: Piñón L; López Y; Núñez E; Ortega M; Pérez MM; Priero A; Rey MJ; Rivas M; Rodríguez CV; Rodríguez JA; Rodríguez RM; Salgado M; Serodio C; Velicia C; Vidal A; Villanueva J;

\section{BIBLIOGRAFÍA}

1. González Merlo J. Ginecología. 7ª ed. Barcelona: Masson; 1997.

2. Instituto Nacional de estadística (INE). Padrón municipal: explotación estadística y Nomenclátor. Madrid: INE; 2009 [actualizada 13 enero 2009; citado 4 junio 2009]. Disponible en:

http://www.ine.es/jaxi/menu.do?type=pcaxis\&path=\%2Ft20\%2Fe245\&file=inebase\&L= $\underline{0}$

3. Cerdá Mota T, Fernández Pampillón ME, Puime Montero P, Lorenzo García V. Enquisa de saúde sanitaria e social das mulleres.Galicia 2000. Santiago de Compostela: Xunta de Galicia. Consellería de Sanidade e Servicios Sociais. Dirección Xeral de Saúde Pública; 2000.

4. Rozenbaum $\mathrm{H}$. ¿Pourquoi la ménopause est-elle devenue un problème de santé publique?. Therapie.1998;53:49-59.

5. WHO. Research on the menoapuse in the 199s. Technical Report Series $n^{0} 888$. Genéve: World Health Organization; 1996.

6. Dennerstein L, Dudley EC, Hopper JL, Guthrie JR, Burger HG. A prospective population-based study of menopausal symptoms. Obstetric Gynecol .2000;96:351-8.

7. Dennerstein L. Quality of life and menopause. Report from the 13th Annual meeting of the North American Menopause Society In: Medscape Ob/Gyn \& Women's Health.2002.

8. García Padilla FM, Toronjo Gómez AM, López Santos V, Contreras Martín A, Toscano Márquez T. Educación para la salud en el climaterio: un consenso sobre sus contenidos. Aten Primaria.1997;20(10):536-42.

9. Lluch E. Experiencias con un grupo de mujeres climátericas. Enf Integral.1992;27:6-9. 
10. Contreras Martín A, Toscano Márquez T, Toronjo Gómez A, Garcia Padilla FM, López Santos V. Las mujeres y la menopausia: ¿qué saben? ¿qué sienten? ¿qué demandan?. Enferm Cient.1997;188-189:13-9.

11. García Padilla F, López Santos V, Toronjo Gómez A, Toscano Márquez T, Contreras Martín A. Valoración de conocimientos sobre el climaterio en mujeres andaluzas. Aten Primaria.2000;26(7):476-81.

12. Arnedillo Sánchez MS, Pozo Pérez F, Zaragoza Pérez MC. Grado de información y actitudes de las mujeres premenopáusicas sobre el climaterio. Matronas prof.2001;5:35-9.

13. Peña Arrébola A. El ejercicio en la prevención y tratamiento de la osteoporosis. Rev osteoporosis en Aten Primaria.1996;2:16-9.

14. López Santos M, García Padilla FM, Toscano Márquez T, Contreras Martín A. Análisis de los estilos de vida de la mujer climatérica: un componente esencial en el estudio de las necesidades educativas. Enferm Clin.2005;15(4):213-9.

15. García Padilla F, López Santos M, Contreras Martín A, Toscano Márquez T, Toronjo Gómez A. Test de conocimientos sobre el climaterio: proceso de construcción y validación. Aten Primaria.2000;26(7):472-5.

16.SENC. (Sociedad Española Nutrición Comunitaria). Guías Alimentarias para la Población Española. Recomendaciones para una dieta saludable. Madrid: IM\&C, S.A.; 2001.

17. Palma Linares I. Habitos alimentarios y actividad física en el tiempo libre de las mujeres adultas catalanas [tesis doctoral]. Barcelona: Departament de Salut Pública, Universitat de Barcelona; 2004.

18. Martín Zurro A, Cano Pérez JF. Atención Primaria: conceptos, organización y práctica. Volumen I. 5a ed. Madrid: Elsevier; 2003.

19.Salas-Salvadó J, Bonada A, Trallero R, Saló MR. Nutrición y Dietética Clínica. Barcelona: Masson; 2000.

20. Álvarez Gómez R, Martín Gramcko E, Bordones de Alvarez M. Conocimiento y actitud sobre el climaterio en mujeres entre 40 y 50 años. Rev Obstet Ginecol Venez .2008;68(1):32-40.

21. Triviño Z, Stiepovich J, Merino J. Factores predictores de conductas promotoras de salud en mujeres peri-post-menopáusicas de Cali, Colombia. Revista Colombia Médica [revista en Internet]. 2007 [citado 22 diciembre 2008];38(4): Disponible en: http://colombiamedica.univalle.edu.co/Vol38No4/html\%20v38n4/v38n4a9.html 
22. Vega M G, Hernandez L A, Leo A G, Vega M J, Escartin Ch M, Luengas M J, et al. Incidencia y factores relacionados con el síndrome climatérico en una población de mujeres mexicanas. Rev Chil Obstet Ginecol.2007 72(5):314-20.

23. Velasco-Murillo V, Fernández-Gárate IH, Ojeda-Mijares RI, Padilla-Vallejo I, de la Cruz-Mejía L. Conocimientos, experiencias y conductas durante el climaterio y la menopausia en las usuarias de los servicios de medicina familiar del IMSS. Rev Med Inst Mex Seguro Soc .2007;45(6):549-56.

24. Enquisa sobre os hábitos alimentarios da poboación adulta galega, 2007. Boletín Epidemiolóxico de Galicia [revista en Internet]. 2008 [citado 12 enero 2009]; XXI(3): Disponible en:

http://www.sergas.es/MostrarContidos N3 T02.aspx?IdPaxina=62485\&uri=http://www .sergas.es/gal/DocumentacionTecnica/docs/SaudePublica/begs/BEG08n3.pdf\&hifr=90 $\underline{0 \& \text { seccion }=0}$

25. Junta de Andalucía. Guía de atención a la salud de la mujer en el climaterio y la madurez. Sevilla: Junta de Andalucía, Consejería de Salud; 2000. 


\section{CUESTIONARIO SOBRE LOS COMPORTAMIENTOS RELACIONADOS CON LA SALUD DE LA MUJER EN EL CLIMATERIO}

FECHA:

$\mathrm{N}^{\circ}$ DE REGISTRO:

CENTRO DE SALUD:

P1 Edad

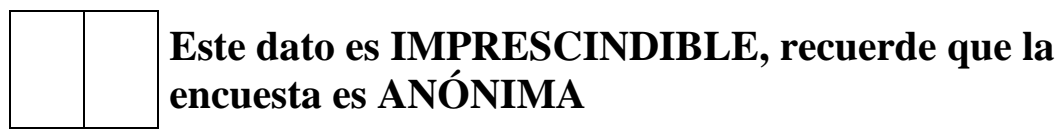

Marque con una $X$ la casilla que más se aproxime a su respuesta

\begin{tabular}{l|l|l}
\hline P2 Zona de residencia & 1 & Rural \\
\cline { 1 - 2 } 2 & Semirural \\
\cline { 1 - 1 } 3 & Urbana
\end{tabular}

P3 Estado civil

\begin{tabular}{|r|l}
\hline 1 & Soltera \\
\cline { 1 - 1 } 2 & Casada \\
\cline { 1 - 1 } 3 & Viuda \\
\cline { 1 - 1 } 4 & Divorciada o separada
\end{tabular}

P4 iVive en pareja?

\begin{tabular}{|l|l|}
\hline 1 & Sí \\
\cline { 1 - 1 } 2 & No \\
\hline
\end{tabular}

P5 Número de hijos

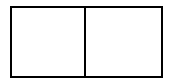

P6 ¿Qué estudios ha realizado?

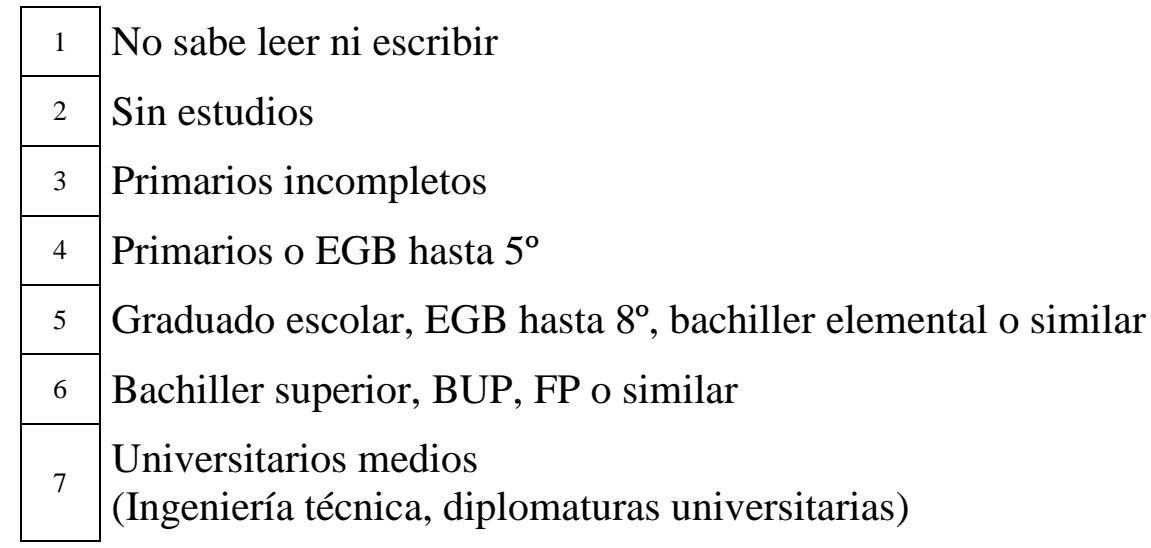




\section{P7 Profesión}

P8 Situación laboral

\begin{tabular}{|c|c|}
\hline 1 & Activa fija \\
\hline 2 & Activa temporal \\
\hline 3 & En paro con subsidio de desempleo \\
\hline 4 & En paro sin subsidio de desempleo \\
\hline 5 & Pensionista \\
\hline 6 & Exclusivamente ama de casa \\
\hline
\end{tabular}

\begin{tabular}{ll|l|l|}
\hline P9 & $\begin{array}{l}\text { ¿Se le ha } \\
\text { retirado la regla? }\end{array}$ & $\begin{array}{l}\text { Sí } \\
\end{array}$ & No
\end{tabular}

P10 ¿Cuánto tiempo hace?

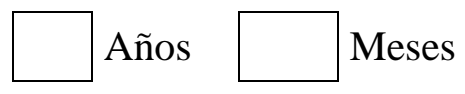

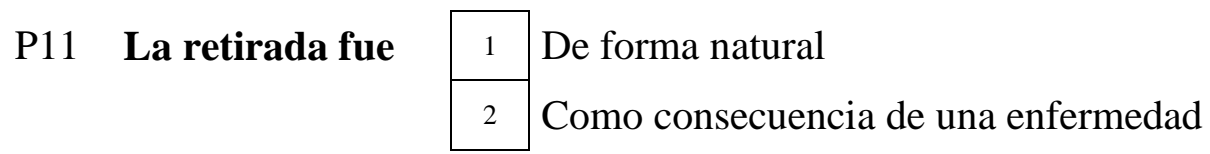

¿Con qué frecuencia toma usted los siguientes alimentos?

\begin{tabular}{|c|c|c|c|c|c|c|}
\hline & & $\begin{array}{c}\text { Más de una vez } \\
\text { al día }\end{array}$ & $\begin{array}{c}\text { Una } \\
\text { vez al } \\
\text { día }\end{array}$ & $\begin{array}{c}\text { Alguna vez a la } \\
\text { semana }\end{array}$ & $\begin{array}{l}\text { Raras } \\
\text { veces }\end{array}$ & Nunca \\
\hline P12 & Queso fresco & 1 & 2 & 3 & 4 & 5 \\
\hline P13 & $\begin{array}{l}\text { Queso curado o } \\
\text { semicurado }\end{array}$ & 1 & 2 & 3 & 4 & 5 \\
\hline P14 & Yoghurt & 1 & 2 & 3 & 4 & 5 \\
\hline P15 & Flan, natillas, etc. & 1 & 2 & 3 & 4 & 5 \\
\hline $\mathrm{PlC}$ & Garbanzos, lentejas & 1 & 2 & 3 & 4 & 5 \\
\hline
\end{tabular}


¿Con qué frecuencia toma usted los siguientes alimentos?

\begin{tabular}{|c|c|c|c|c|c|c|}
\hline P17 & \begin{tabular}{|l} 
Zanahorias, lechuga, \\
alcachofas, calabacín, \\
cebolla, coles, berza, \\
brécol, berros o puerros.
\end{tabular} & 1 & 2 & 3 & 4 & 5 \\
\hline P18 & $\begin{array}{l}\text { Otras verduras (tomates, } \\
\text { judías verdes, acelgas, } \\
\text { espinacas, etc.) }\end{array}$ & 1 & 2 & 3 & 4 & 5 \\
\hline P19 & Kiwis y ciruelas & 1 & 2 & 3 & 4 & 5 \\
\hline $\mathrm{P} 20$ & $\begin{array}{l}\text { Otras frutas (naranjas, } \\
\text { fresas, melón, etc.) }\end{array}$ & 1 & 2 & 3 & 4 & 5 \\
\hline $\mathrm{P} 21$ & Frutos secos & 1 & 2 & 3 & 4 & 5 \\
\hline $\mathrm{P} 22$ & $\begin{array}{l}\text { Gambas, mejillones, } \\
\text { almejas o pulpo }\end{array}$ & 1 & 2 & 3 & 4 & 5 \\
\hline $\mathrm{P} 23$ & Pescado azul & 1 & 2 & 3 & 4 & 5 \\
\hline $\mathrm{P} 24$ & Pescado blanco & 1 & 2 & 3 & 4 & 5 \\
\hline $\mathrm{P} 25$ & Chocos/calamares & 1 & 2 & 3 & 4 & 5 \\
\hline $\mathrm{P} 26$ & Carne de ave & 1 & 2 & 3 & 4 & 5 \\
\hline $\mathrm{P} 27$ & $\begin{array}{l}\text { Carne de ternera, } \\
\text { cordero... }\end{array}$ & 1 & 2 & 3 & 4 & 5 \\
\hline $\mathrm{P} 28$ & Carne de cerdo. & 1 & 2 & 3 & 4 & 5 \\
\hline P29 & Embutidos & 1 & 2 & 3 & 4 & 5 \\
\hline P30 & Huevos & 1 & 2 & 3 & 4 & 5 \\
\hline P31 & $\begin{array}{l}\text { Cereales (arroz, } \\
\text { pastas, maíz...) }\end{array}$ & 1 & 2 & 3 & 4 & 5 \\
\hline
\end{tabular}


¿Con qué frecuencia toma usted los siguientes alimentos?

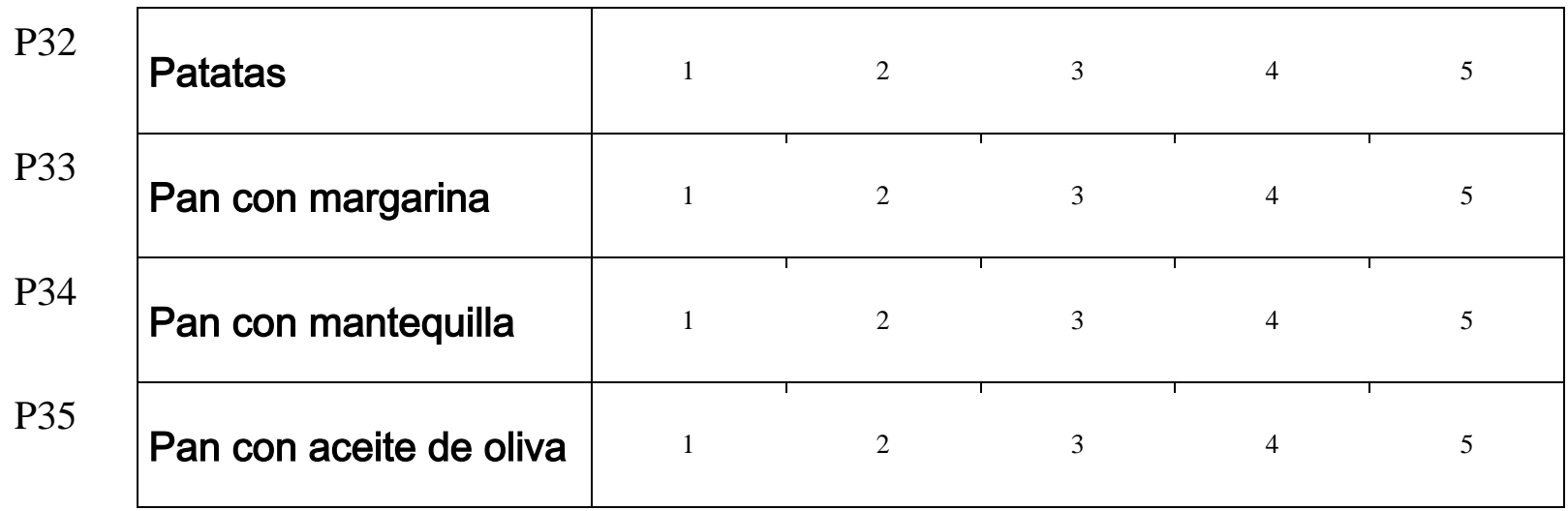

P36 ¿Cómo suele tomar los alimentos?

\begin{tabular}{|c|c|}
\hline & Sin sal \\
\hline & Con poca sal \\
\hline & Bien sazonados \\
\hline
\end{tabular}

P37 La leche que toma habitualmente es:

\begin{tabular}{|c|c|}
\hline 1 & Entera \\
\hline 2 & Desnatada \\
\hline 3 & Semidesnatada \\
\hline 4 & En polvo \\
\hline 5 & Condensada \\
\hline 6 & No tomo leche (Pasa a la pregunta 39$)$ \\
\hline
\end{tabular}

P38 ¿Qué cantidad de leche suele tomar al día?

\begin{tabular}{|c|c|}
\hline 1 & Más de tres vasos \\
\hline 2 & Dos o tres vasos \\
\hline 3 & Un vaso \\
\hline 4 & Menos de un vaso \\
\hline 5 & No la tomo en forma liquida \\
\hline
\end{tabular}

P39 ¿Qué cantidad de pan suele tomar al día?

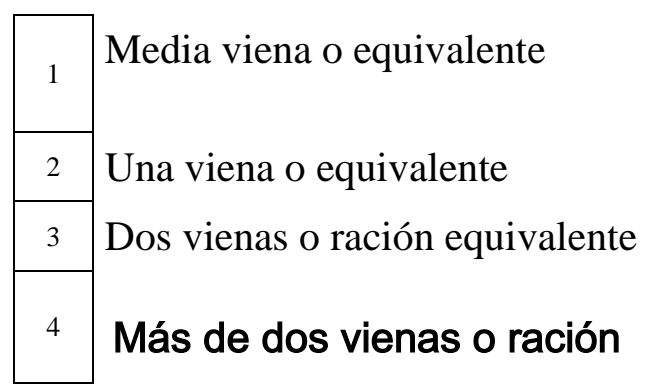




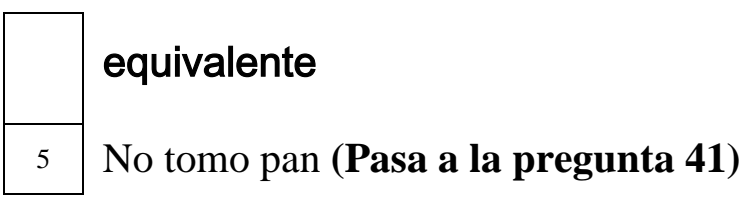

\begin{tabular}{|c|c|}
\hline 1 & Pan blanco \\
\hline 2 & Pan integral \\
\hline & Tomo indistintamente \\
\hline
\end{tabular}

P41 ¿Con qué frecuencia suele tomar dulces o pasteles?

\begin{tabular}{|c|c|}
\hline 1 & Diariamente \\
\hline 2 & Dos o tres veces por semana \\
\hline 3 & Una vez por semana \\
\hline 4 & $\begin{array}{l}\text { De forma esporádica (de vez en } \\
\text { cuando) }\end{array}$ \\
\hline 5 & $\begin{array}{l}\text { Nunca los tomo (Pasa a la pregunta } \\
\text { 45) }\end{array}$ \\
\hline
\end{tabular}

\begin{tabular}{|c|l}
\hline 1 & Mucha cantidad \\
\hline 2 & Bastante \\
\hline 3 & Poca cantidad \\
\cline { 1 - 1 } 4 & Muy poca \\
\hline
\end{tabular}

P43 Cuando toma dulces o pasteles, suele hacerlo:

\begin{tabular}{|c|l}
\hline 1 & Después de las comidas \\
\cline { 1 - 1 } 2 & Entre comidas \\
\cline { 1 - 1 } 3 & Ambas cosas \\
\hline
\end{tabular}

\begin{tabular}{|c|c|}
\hline & Caseros \\
\hline & De Pastelerías \\
\hline & Bollería Industr \\
\hline
\end{tabular}


¿Con qué frecuencia suele tomar alimentos preparados de la siguiente forma:?

\begin{tabular}{l|l|c|c|c|c|c|}
\cline { 3 - 7 } & & $\begin{array}{c}\text { Con mucha } \\
\text { frecuencia }\end{array}$ & $\begin{array}{c}\text { Con bastante } \\
\text { frecuencia }\end{array}$ & $\begin{array}{c}\text { Con una } \\
\text { frecuencia } \\
\text { moderada }\end{array}$ & $\begin{array}{c}\text { Con poca } \\
\text { Frecuencia }\end{array}$ & $\begin{array}{c}\text { No suelo } \\
\text { tomarlos de } \\
\text { esta forma }\end{array}$ \\
\cline { 2 - 7 } P45 & Fritos & 1 & 2 & 3 & 4 & 5 \\
P46 & Cocidos & 1 & 2 & 3 & 4 & 5 \\
P47 & Asados & 1 & 2 & 3 & 4 & 5 \\
\hline P48 & En conserva & 1 & 2 & 3 & 4 & 5 \\
\hline P49 & Precocinados & 1 & 3 & 4 & 5 \\
\hline P50 & Crudos (verduras y frutas) & 1 & 2 & 3 & 4 & 5 \\
\hline P51 & Picantes & 1 & 2 & 3 & 4 & 5 \\
\hline P52 & Muy calientes & 1 & 2 & 3 & 4 & 5 \\
\hline
\end{tabular}

P53 En su casa, ¿qué tipo de grasa se suele utilizar habitualmente para cocinar?:

\begin{tabular}{|c|c|}
\hline 1 & Aceite de oliva \\
\hline 2 & Aceite de Girasol \\
\hline 3 & Mantequilla o margarina \\
\hline & Otras \\
\hline
\end{tabular}

¿Con qué frecuencia toma las siguientes bebidas?

\begin{tabular}{|c|c|c|c|c|c|c|c|}
\hline & & $\begin{array}{c}\text { Más de tres } \\
\text { veces al } \\
\text { día }\end{array}$ & $\begin{array}{l}\text { De dos a } \\
\text { tres veces }\end{array}$ & $\begin{array}{c}\text { Una } \\
\text { vez } \\
\text { al día }\end{array}$ & $\begin{array}{c}\text { Varias } \\
\text { veces por } \\
\text { semana }\end{array}$ & $\begin{array}{l}\text { Raras } \\
\text { veces }\end{array}$ & Nunca \\
\hline P54 & Un vaso de cerveza/sidra & 1 & 2 & 3 & 4 & 5 & 6 \\
\hline P55 & Un vaso de vino & 1 & 2 & 3 & 4 & 5 & 6 \\
\hline P56 & Una copa de licor & 1 & 2 & 3 & 4 & 5 & 6 \\
\hline P57 & Una copa de ginebra, ron, etc. & 1 & 2 & 3 & 4 & 5 & 6 \\
\hline P58 & Un vaso de bebida gaseosa & 1 & 2 & 3 & 4 & 5 & 6 \\
\hline P59 & Un vaso de zumo natural & 1 & 2 & 3 & 4 & 5 & 6 \\
\hline P60 & Una taza de café o té & 1 & 2 & 3 & 4 & 5 & 6 \\
\hline
\end{tabular}

¿Qué cantidad de agua suele tomar diariamente?

\begin{tabular}{l|l|c|c|c|c|c|}
\cline { 3 - 7 } \multicolumn{2}{l|}{} & $\begin{array}{c}250 \mathrm{cc} \text { (un vaso) o } \\
\text { menos }\end{array}$ & $500 \mathrm{cc}$ & $1000-1500 \mathrm{cc}$ & $1500-2000 \mathrm{cc}$ & Más de 2000 \\
\cline { 2 - 7 } P61 & En verano & 1 & 2 & 3 & 4 & 5 \\
\cline { 2 - 7 } P62 & Durante el resto del año & 1 & 2 & 3 & 4 & 5 \\
\hline
\end{tabular}


Habitualmente, ¿cómo suele hacer las siguientes comidas?

\begin{tabular}{l|l|c|c|c|}
\cline { 2 - 5 } & & & & \\
& & & & \\
\end{tabular}

P69 Su situación respecto al tabaco es

\begin{tabular}{|c|c|}
\hline 1 & No ha fumado nunca \\
\hline 2 & Fumaba pero lo dejo hace más de un año \\
\hline 3 & Fumaba pero lo dejo hace menos de un año \\
\hline 4 & Esta intentando dejar de fumar \\
\hline 5 & Fuma \\
\hline
\end{tabular}

P70 Si es usted fumadora habitual, ¿cuántos cigarrillos suele fumarse al día?

\begin{tabular}{|c|}
\hline \\
\hline \multirow{2}{*}{2 De 6 a 10} \\
\hline \\
\hline \\
\hline De 1 a 5 \\
\hline \\
\hline De 11 a 20 \\
\hline Más de \\
\hline
\end{tabular}

MUCHAS GRACIAS POR SU COLABORACIÓN 


\section{TEST DE CONOCIMIENTO SOBRE EL CLIMATERIO MUJERES DE 30 A 60 AÑOS}

RESPUESTAS

1. Se dice que una mujer tiene la menopausia cuando lleva seis meses sin tener la regla.

2. El ejercicio físico durante la menopausia provoca un desgaste en los huesos y articulaciones.

3.La relación de la mujer con su pareja, familiares, amigos, influye en el modo en que ésta acepta la menopausia.

4.Se dice que una mujer tiene una menopausia prematura o precoz cuando, se le retira la regla antes de los 40 años.

5.Muchas mujeres disfrutan más de las relaciones sexuales a partir de la menopausia al desaparecer el riesgo de embarazo.

6.Cuando los hijos se independizan, muchas mujeres piensan que su función en la vida se ha terminado.

7.Hay mujeres que viven la desaparición definitiva de la regla como una liberación.

8.Es perjudicial para la salud mantener relaciones sexuales durante la menopausia.

9 El climaterio es una etapa de la vida de la mujer que engloba a la menopausia.

10.La marcha de los hijos del hogar y la llegada de los nietos no influye en la vivencia de la menopausia.

11 La mayoría de las mujeres a partir de la menopausia se sienten más incomprendidas.

12.La osteoporosis consiste en un aumento de la superficie del hueso

13.Es recomendable tomar más calcio durante la menopausia, sobre todo, si con anterioridad se ha tomado poca cantidad.

14.Los medicamentos son imprescindibles para tratar los problemas de sueño que aparecen durante la menopausia.

15.Todas las mujeres presentan los mismos síntomas durante la menopausia.

16.Los sofocos y sudores que suceden durante la menopausia son achacables al nerviosismo de las mujeres.

17.La fibra, necesaria para una alimentación adecuada, se encuentra principalmente en la leche y sus derivados.

18.La sequedad vaginal es un síntoma que puede aparecer en la menopausia.

19.Durante la menopausia los ovarios reducen la producción de estrógenos.

20.Comentar los cambios de la menopausia y conocer sus causas ayuda a tener una actitud positiva ante la misma.

21.Al retirarse la regla, la mujer no necesita tomar alimentos ricos en hierro.

22.Cuando la realización del coito provoca molestias debería evitarse la actividad sexual.

23.Las mujeres que han tenido una menopausia precoz están más protegidas contra las enfermedades del corazón y de la circulación.

24.El tratamiento hormonal es poco recomendable en la mujer con menopausia precoz.

25.Todos los tratamientos hormonales se presentan en forma de parches.

26.Las mujeres con una personalidad formada afrontan mejor la menopausia.

27.La mujer durante la menopausia debe tomar menos agua para evitar la subida de tensión arterial.

28.Nuestra sociedad considera a la mujer menopaúsica algo neurótica.

29.La disminución de los estrógenos hace que la vagina pierda parte de su elasticidad.

30.La osteoporosis aparece con igual frecuencia en hombres y mujeres de edad avanzada.

31.El tipo de vida que haya tenido la mujer en etapas anteriores influye en la vivencia de la menopausia.

$\mathrm{V}-\mathrm{F} \quad \mathrm{ns} / \mathrm{nc}$

$\mathrm{V}-\mathrm{F}$ ns/nc

$\mathrm{V}-\mathrm{F} \quad \mathrm{ns} / \mathrm{nc}$

$\mathrm{V}-\mathrm{F} \quad \mathrm{ns} / \mathrm{nc}$

$\mathrm{V}-\mathrm{F} \quad \mathrm{ns} / \mathrm{nc}$

V - F ns/nc

$\begin{array}{ll}\mathrm{V}-\mathrm{F} & \mathrm{ns} / \mathrm{nc}\end{array}$

$\mathrm{V}-\mathrm{F} \quad \mathrm{ns} / \mathrm{nc}$

$\mathrm{V}-\mathrm{F} \quad \mathrm{ns} / \mathrm{nc}$

$\mathrm{V}-\mathrm{F} \quad \mathrm{ns} / \mathrm{nc}$

$\mathrm{V}-\mathrm{F} \quad \mathrm{ns} / \mathrm{nc}$

V - F ns/nc

$\mathrm{V}-\mathrm{F}$ ns/nc

\begin{tabular}{l|l}
$\mathrm{V}-\mathrm{F}$ & $\mathrm{ns} / \mathrm{nc}$
\end{tabular}

V - F ns/nc

$\mathrm{V}-\mathrm{F} \quad \mathrm{ns} / \mathrm{nc}$

F

$\mathrm{V}-\mathrm{F} \quad \mathrm{ns} / \mathrm{nc}$

$\mathrm{V}-\mathrm{F} \quad \mathrm{ns} / \mathrm{nc}$

$\mathrm{V}$ - F ns/nc

$\begin{array}{ll}\mathrm{V}-\mathrm{F} & \mathrm{ns} / \mathrm{nc}\end{array}$

$\begin{array}{ll}\mathrm{V}-\mathrm{F} & \mathrm{ns} / \mathrm{nc}\end{array}$

$\mathrm{V}-\mathrm{F} \quad \mathrm{ns} / \mathrm{nc}$

V - F

$\begin{array}{ll}\mathrm{V}-\mathrm{F} & \mathrm{ns} / \mathrm{nc}\end{array}$

$\begin{array}{ll}\mathrm{V}-\mathrm{F} & \mathrm{ns} / \mathrm{nc}\end{array}$

$\begin{array}{lll}\mathrm{V}-\mathrm{F} & \mathrm{ns} / \mathrm{nc}\end{array}$

$\mathrm{V}-\mathrm{F}$ ns/nc

$\mathrm{V}-\mathrm{F}$ ns/nc

$\mathrm{V}-\mathrm{F}$ ns/nc

\begin{tabular}{l|l}
$\mathrm{V}-\mathrm{F}$ & $\mathrm{ns} / \mathrm{nc}$
\end{tabular}

\begin{tabular}{c|c}
$\mathrm{V}-\mathrm{F}$ & $\mathrm{ns} / \mathrm{nc}$ \\
$\mathrm{V}-\mathrm{F}$ &
\end{tabular}

$\begin{array}{ll}\mathrm{V}-\mathrm{F} & \mathrm{ns} / \mathrm{nc}\end{array}$ 


\begin{tabular}{|c|c|c|}
\hline 32.A partir de la menopausia la mujer necesita una dieta rica en calorías. & $\mathrm{V}-\mathrm{F}$ & $\mathrm{ns} / \mathrm{nc}$ \\
\hline $\begin{array}{l}\text { 33. No se debe reducir la cantidad de azúcar que se toma durante la menopausia porque es buena para los problemas } \\
\text { de huesos. }\end{array}$ & $\mathrm{V}-\mathrm{F}$ & $\mathrm{ns} / \mathrm{nc}$ \\
\hline 34.A partir de la menopausia la mujer deja de tener la regla, pero sigue teniendo la ovulación & $\mathrm{V}-\mathrm{F}$ & $\mathrm{ns} / \mathrm{nc}$ \\
\hline 35.La menopausia quirúrgica o artificial es aquella que aparece a partir de la ligadura de trompas. & $\mathrm{V}-\mathrm{F}$ & $\mathrm{ns} / \mathrm{nc}$ \\
\hline $\begin{array}{l}\text { 36.La mujer debe realizar ejercicios intensos que le ayude en poco tiempo a mejorar los problemas que aparecen en } \\
\text { la menopausia. }\end{array}$ & $\mathrm{V}-\mathrm{F}$ & $\mathrm{ns} / \mathrm{nc}$ \\
\hline 37.La vivencia del climaterio, o etapa que rodea a la menopausia, es igual en todos los países y culturas. & $\mathrm{V}-\mathrm{F}$ & $\mathrm{ns} / \mathrm{nc}$ \\
\hline 38.La retirada de la regla a los 50 años se considera una menopausia tardía. & $\mathrm{V}-\mathrm{F}$ & $\mathrm{ns} / \mathrm{nc}$ \\
\hline 39.Las mujeres tienen más riesgo de fractura de hueso a partir de la menopausia. & $\mathrm{V}-\mathrm{F}$ & $\mathrm{ns} / \mathrm{nc}$ \\
\hline $\begin{array}{l}\text { 40.El proceso de adaptación al climaterio es más difícil cuando coincide con cambios familiares y laborales } \\
\text { importantes. }\end{array}$ & $\mathrm{V}-\mathrm{F}$ & $\mathrm{ns} / \mathrm{nc}$ \\
\hline $\begin{array}{l}\text { 41Los tratamientos no hormonales, tienen la función de regular el nivel de estrógenos en el organismo en la etapa } \\
\text { menopaúsica de la mujer. }\end{array}$ & $\mathrm{V}-\mathrm{F}$ & $\mathrm{ns} / \mathrm{nc}$ \\
\hline 42.El método anticonceptivo elegido deber ser el mismo a lo largo de toda la etapa fértil de la mujer. & $\mathrm{V}-\mathrm{F}$ & $\mathrm{ns} / \mathrm{nc}$ \\
\hline 43.La mujer menopaúsica es una mujer incompleta. & $\mathrm{V}-\mathrm{F}$ & $\mathrm{ns} / \mathrm{nc}$ \\
\hline 44. Durante el período que precede a la menopausia no cambia la duración habitual del ciclo menstrual. & $\mathrm{V}-\mathrm{F}$ & $\mathrm{ns} / \mathrm{nc}$ \\
\hline 45.La actividad sexual puede acelerar los cambios que aparecen en los órganos genitales durante la menopausia. & $\mathrm{V}-\mathrm{F}$ & $\mathrm{ns} / \mathrm{nc}$ \\
\hline 46. La disminución de estrógenos durante la menopausia favorece la circulación sanguínea. & $\mathrm{V}-\mathrm{F}$ & $\mathrm{ns} / \mathrm{nc}$ \\
\hline $\begin{array}{l}\text { 47.Las tareas que realiza habitualmente el ama de casa son un buen ejercicio para mejorar algunos de los problemas } \\
\text { de huesos que pueden aparecer. }\end{array}$ & $V-F$ & $\mathrm{~ns} / \mathrm{nc}$ \\
\hline 48.Muchas mujeres temen que la menopausia las haga menos atractivas sexualmente. & $\mathrm{V}-\mathrm{F}$ & $\mathrm{ns} / \mathrm{nc}$ \\
\hline $\begin{array}{l}\text { 49.La gimnasia de mantenimiento y los paseos al aire libre son los únicos ejercicios recomendados durante la } \\
\text { menopausia. }\end{array}$ & $\mathrm{V}-\mathrm{F}$ & $\mathrm{ns} / \mathrm{nc}$ \\
\hline 50.Las mujeres con tratamiento hormonal están obligadas a acudir a revisiones médicas periódicamente. & $\mathrm{V}-\mathrm{F}$ & $\mathrm{ns} / \mathrm{nc}$ \\
\hline 51.Mantener actividades fuera del hogar, ayuda a vivir mejor la llegada de la menopausia. & $\mathrm{V}-\mathrm{F}$ & $\mathrm{ns} / \mathrm{nc}$ \\
\hline 52.Todas las mujeres pueden ponerse los parches de estrógenos. & $\mathrm{V}-\mathrm{F}$ & $\mathrm{ns} / \mathrm{nc}$ \\
\hline 53.El que en nuestro entorno se relacione la menopausia con la vejez hace que se viva peor esta etapa. & $\mathrm{V}-\mathrm{F}$ & $\mathrm{ns} / \mathrm{nc}$ \\
\hline $\begin{array}{l}\text { 54.La disminución de estrógenos que se producen con la menopausia protege a la mujer de las enfermedades } \\
\text { cardiovasculares. }\end{array}$ & $\mathrm{V}-\mathrm{F}$ & $\mathrm{ns} / \mathrm{nc}$ \\
\hline 55.Las mujeres delgadas suelen tener menos molestias relacionadas con la menopausia. & $\mathrm{V}-\mathrm{F}$ & $\mathrm{ns} / \mathrm{nc}$ \\
\hline 56.Sólo cuando aparecen hemorragias es necesario ir & $\mathrm{V}-\mathrm{F}$ & $\mathrm{ns} / \mathrm{nc}$ \\
\hline
\end{tabular}

ISSN 1695-6141 